\title{
Ultrasonido pulmonar en pacientes con COVID-19: revisión sistemática
}

\section{Lung ultrasound in patients with COVID-19: Sistematic review}

\author{
Karen E. Fernández-Rojas y Graciela Merinos-Sánchez* \\ Servicio de Urgencias de Adultos, Hospital General de México "Dr. Eduardo Liceaga", Secretaría de Salud, Ciudad de México, México
}

\begin{abstract}
Resumen
Objetivo: Establecer la utilidad del ultrasonido pulmonar en los pacientes con COVID-19. Material y métodos: Se trata de una revisión sistemática según PRISMA de EQUATOR, con búsqueda en las principales bases mundiales de datos: PubMed, Scopus (Elsevier), Epistemónikos, EMBASE y Europe PMC. Resultados: Se encontraron 2,318 artículos; después del escrutinio por parte de los autores, se seleccionaron 15 publicaciones, de las que se extrajo la información para el análisis y la revisión sistemática narrativa. Conclusiones: El ultrasonido pulmonar puede desempeñar un papel importante en el diagnóstico y pronóstico de las lesiones pulmonares causadas por enfermedad por coronavirus 2019 (COVID-19), ayuda en la toma de decisiones clínicas a los médicos de primera línea que atienden pacientes con enfermedad respiratoria aguda por coronavirus.
\end{abstract}

Palabras clave: Medicina de Emergencia. Coronavirus. Ultrasonografía.

\begin{abstract}
Objective: To establish the usefulness of pulmonary ultrasound in patients with COVID-19. Material and methods: This is a systematic review according to PRISMA of EQUATOR, with a search PubMed, Scopus (Elsevier), Epistemónikos, EMBASE and Europe PMC. Results: 2318 articles were found, after scrutiny by the authors, 15 publications were selected, from which the information was extracted for analysis and narrative systematic review. Conclusions: Lung ultrasound can play an important role in the diagnosis and prognosis of lung lesions caused by COVID-19, it helps first-line doctors who care for patients with acute respiratory disease due to Coronavirus in clinical decision-making.
\end{abstract}

Key words: Emergency Medicine. Coronavirus. Ultrasonography.

\section{Introducción}

El 31 de diciembre de 2019, China informó a la Organización Mundial de la Salud (OMS) de varios casos de neumonía de etiología desconocida detectados en la ciudad de Wuhan, provincia de Hubei. Posteriormente se descubrió que esta nueva enfermedad era causada por el coronavirus 2 del síndrome respiratorio agudo grave (SARS-CoV-2) y la denominaron enfermedad por coronavirus 2019 (COVID-19) ${ }^{1}$. Hoy en día es una pandemia. Al 5 de agosto del 2020 había $9,741,727$ casos y 365,334 muertos en el mundo, mientras que en México, según la Secretaría de Salud y la Dirección General de Epidemiología se reportaban 456,100 casos de infectados acumulados y 49,698
Correspondencia:

*Graciela Merinos-Sánchez

E-mail: grace25ms@ hotmail.com
Disponible en internet: 16-07-2021 Rev Hosp Jua Mex. 2021;88(3):122-129

www.revistahospitaljuarez.com 1405-9622/@ 2021 Sociedad Médico-Quirúrgica del Hospital Juárez de México, A.C. Publicado por Permanyer. Este es un artículo open access bajo la licencia CC BY-NC-ND (http://creativecommons.org/licenses/by-nc-nd/4.0/). 
muertes, siendo entonces el tercer país del mundo con más muertes y el sexto mundial en número de contagios por el virus ${ }^{1-3}$.

Los signos y síntomas clínicos del virus en cuestión son odinofagia, disfagia, ojos rojos, fiebre, tos seca, artralgias y mialgias; los casos graves desarrollan dificultad respiratoria por lesiones inflamatorias pulmonares $^{3,4}$. La COVID-19 afecta a otros órganos, pero de los más afectados son los pulmones, donde el virus ingresa a las células del huésped a través de la proteína del receptor de membrana plasmática, enzima convertidora de angiotensina 2 , expresada más abundantemente en las células alveolares tipo II de los pulmones $^{5}$. En relación con lo dicho anteriormente, la afectación pulmonar provocada por este virus es especialmente preocupante, debido a la presencia del síndrome de dificultad respiratoria aguda (SDRA) y a la facilidad para generar neumonía ${ }^{6}$, por lo que muchos trabajadores de la salud han optado por el ultrasonido pulmonar (USP) en los casos sospechosos de COVID19, tanto para la evaluación inicial como para el seguimiento $^{6-8}$. En estos estudios destaca el trabajo multidisciplinario, sin embargo, parece que en México el USP es una competencia limitada a pocas especialidades.

La ultrasonografía es una técnica de imagen que se considera como un método confiable, no invasivo de cabecera, para una detección y localización precisa de la enfermedad, por lo que es de gran utilidad en los pacientes críticos que se encuentran en el servicio de urgencias o una unidad de cuidados intensivos ${ }^{9}$. Las características que han convertido esta técnica en un procedimiento de primera línea en los servicios que manejan pacientes en estado crítico son: la ausencia de emisión de radiaciones, lo que la hace apropiada para determinados grupos (niños, mujeres embarazadas), permitiendo repetir la prueba tantas veces sea necesaria sin incrementar el riesgo a la exposición; y la portabilidad a la cabecera del paciente, lo que supone que se evitarían riesgos en los traslados ${ }^{10}$.

Las continuas exploraciones con el USP permiten la comparación directa con los hallazgos encontrados en la radiografía de tórax, debido a la generación de patrones estandarizados y reproducibles (hallazgos similares en términos de ubicación de las áreas de afectación pulmonar y el patrón de la enfermedad parenquimatosa) $)^{4,11,12}$. El pulmón está a expensas de enfermedades bacterianas y, como en este caso, virales, que llegan a complicar y se convierten en neumonía, la cual, de acuerdo con la OMS, es una de las principales causas de muerte en todo el mundo e incluso con mayor recurrencia en los países de bajos ingresos. Por otra parte estas muertes pueden prevenirse mediante la detección temprana y terapia dirigida $^{13}$, sin embargo, el diagnóstico de neumonía no siempre es sencillo ${ }^{14-16}$. Así mismo, la neumonía puede estar asociada al SDRA ${ }^{17}$; hoy en día para poder diagnosticar y tratar en una unidad de cuidados intensivos o servicios de urgencias el padecimiento en cuestión se ha utilizado eficazmente el llamado protocolo BLUE, que utiliza como herramienta principal el USP 14,18,19. En recientes publicaciones de hallazgos claves en el USP para orientar al diagnóstico de neumonía por COVID-19 se ha encontrado que los pacientes presentan lesiones en el campo pulmonar posterior e inferior, además de alteración en la línea pleural y presencia de patrón B en las zonas pulmonares estudiadas 17,20-22.

\section{Pregunta de la revisión}

¿El USP tiene utilidad en el abordaje diagnóstico de pacientes con enfermedad respiratoria aguda por COVID-19?

\section{Material y métodos}

En esta revisión se realizó una búsqueda sistemática en las bases: PubMed, Europe PMC, Epistemónikos y Scopus utilizando las palabras clave en inglés y español: coronavirus, COVID-19, SARS-CoV-2, 2019 nCov y ultrasonido (sonografía y ecografía) pulmonar, con los operadores booleanos AND y OR respectivamente; se descartaron estudios en modelos anatómicos, animales, pacientes pediátricos y embarazadas con el booleano NOT. Con lo anterior se localizaron artículos en inglés o español, Open Access (gratuitos) o con acceso mediante la Biblioteca Médica Digital (http://www.facmed.unam. $\mathrm{mx} / \mathrm{bmd} /$ ) de la Facultad de Medicina de la Universidad Nacional Autónoma de México, mismos que se filtraron con las herramientas de búsqueda avanzada para eliminar aquellos que no contaran con resumen completo, así como revisiones bibliográficas, cartas científicas, cartas al editor, reportes de caso y puntos de vista. Por la poca información disponible, se incluyeron revisiones sistemáticas con o sin metaanálisis, estudios de casos y controles y cohortes. Con lo anterior, los investigadores revisaron los títulos para eliminar artículos que no cumplieran con el objetivo de la revisión; de los títulos elegibles, se revisaron los resúmenes para eliminar los artículos que no cumplieran con el objetivo de la revisión. 


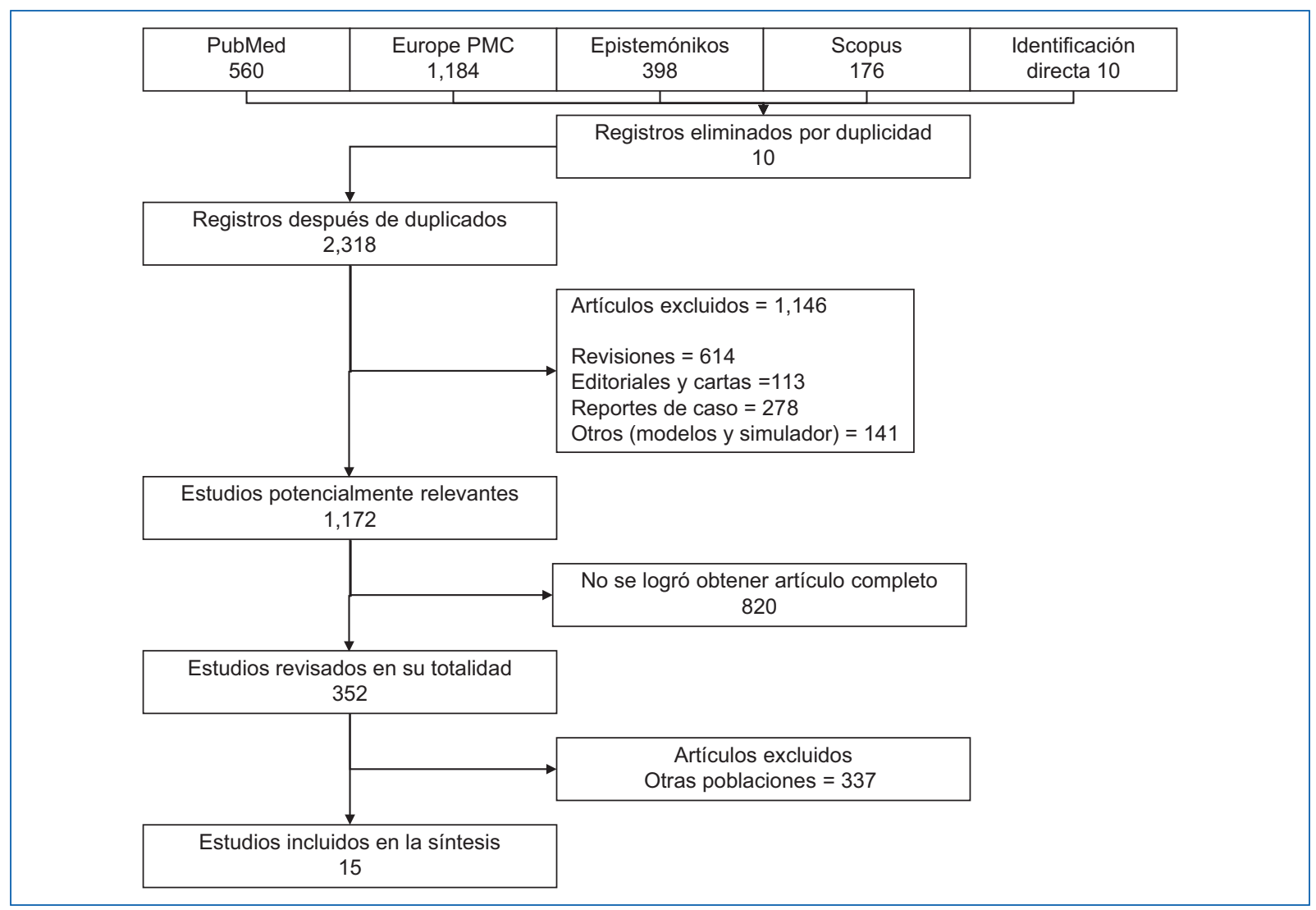

Figura 1. Diagrama PRISMA (Preferred Reporting Items for Systematic reviews and Meta-Analyses) del proceso de selección de los estudios encontrados, eliminados e incluidos en el estudio.

\section{Resultados}

Se realizó la búsqueda según lo previsto en la metodología, se identificaron 2,318 estudios. Después de eliminar estudios duplicados, no realizados en humanos, sin controles, sin resumen disponible ni texto completo en español o inglés, se seleccionaron 15 publicaciones (Fig. 1), de las cuales se extrajo la información de la tabla 17,8,20-32, donde se describe el número de pacientes, el comparador (radiografía y/o tomografía computarizada [TC] de tórax) y los hallazgos principales de cada estudio (Figs. 2 y 3 ).

\section{Discusión}

El USP es una herramienta útil en un servicio de urgencias, gracias a la fácil adquisición de imágenes pleuro-pulmonares que generan información para ayudar al diagnóstico, a la toma de decisiones clínicas y el manejo de insuficiencia respiratoria y lesión pulmonar en pacientes con SARS-CoV-2. Esto se ve poco a poco mejorado por el establecimiento de las características ultrasonográficas de las lesiones pulmonares durante la progresión y recuperación de la enfermedad en cuestión ${ }^{33}$. En el USP se encuentran irregularidades (fractura, interrupción, desniveles y engrosamiento) en la línea pleural, líneas B y patrón B, causados por inflamación y ocupación alveolo-intersticial $^{34}$. Puede ser de utilidad para la detección de pequeñas consolidaciones, de difícil visualización por radiografía de tórax, particularmente cuando el USP se realiza de forma seriada ${ }^{35}$.

En esta revisión sistemática fueron seleccionadas publicaciones donde se comparó el ultrasonido frente a otro método diagnóstico en estudios que incluyeron a 529 pacientes. Evans, Zieleskiewic y Tan señalan en sus estudios que el USP puede ser una alternativa viable para el diagnóstico y la monitorización de la COVID-19, en comparación con la radiografía de tórax, por contar con potencial para mantener una exposición mínima al personal y pacientes al eliminar la necesidad de transporte del paciente, así como evitar la necesidad de radiación 
Tabla 1. Resumen de los resultados por estudio, se enlista autor (año), número de pacientes, comparador, desenlace y conclusión de cada publicación

\begin{tabular}{|c|c|c|c|c|c|c|}
\hline Autor, año & $\begin{array}{l}\text { Tipo de } \\
\text { estudio }\end{array}$ & Pacientes & Comparados & Desenlace & Conclusión & ָั \\
\hline Tan, et al., $2020^{7}$ & $\begin{array}{l}\text { Reporte } \\
\text { prospectivo y } \\
\text { series de } \\
\text { casos }\end{array}$ & 12 & $\begin{array}{l}\text { USP vs. } \\
\text { radiografía y } \\
\text { TC }\end{array}$ & $\begin{array}{l}\text { El USP mostró engrosamiento } \\
\text { pleural, línea pleural discontinua, } \\
\text { línea B parcialmente difusa, signo } \\
\text { de cascada. Las diferencias de las } \\
\text { características ultrasónicas, incluido } \\
\text { el rango de lesión, los signos } \\
\text { pulmonares y las complicaciones } \\
\text { relacionadas con la neumonía entre } \\
\text { el USP y la radiografía fueron } \\
\text { estadísticamente significativas }\end{array}$ & $\begin{array}{l}\text { EI USP es una opción } \\
\text { efectiva para la evaluación } \\
\text { de anomalías pulmonares } \\
\text { durante la epidemia de } \\
\text { COVID-19 }\end{array}$ & 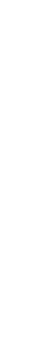 \\
\hline Cho, et al., $2020^{8}$ & $\begin{array}{l}\text { Reporte } \\
\text { prospectivo y } \\
\text { series de } \\
\text { casos }\end{array}$ & 6 & $\begin{array}{l}\text { USP vS. } \\
\text { radiografía }\end{array}$ & $\begin{array}{l}\text { El USP detectó hallazgos anormales } \\
\text { tempranos de líneas B } \\
\text { representativas en un paciente con } \\
\text { una radiografía de tórax normal }\end{array}$ & $\begin{array}{l}\text { El USP es superior que la } \\
\text { radiografía de tórax para } \\
\text { el diagnóstico de } \\
\text { alteraciones asociadas a } \\
\text { la COVID-19 }\end{array}$ & 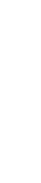 \\
\hline $\begin{array}{l}\text { Evans, et al., } \\
2020^{20}\end{array}$ & $\begin{array}{l}\text { Reporte } \\
\text { prospectivo y } \\
\text { series de } \\
\text { casos }\end{array}$ & 21 & $\begin{array}{l}\text { USP vs. } \\
\text { radiografía }\end{array}$ & $\begin{array}{l}\text { EI USP mostró datos tempranos de } \\
\text { cambios pleurales y líneas o perfil } \\
\text { B; la radiografía no revela datos } \\
\text { pleurales tempranos }\end{array}$ & $\begin{array}{l}\text { El USP es superior a la } \\
\text { radiografía en casos leves } \\
\text { y moderados, así como } \\
\text { herramienta de pronóstico }\end{array}$ & 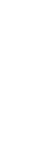 \\
\hline $\begin{array}{l}\text { Guarracino, } \\
\text { et al., } 2020^{21}\end{array}$ & $\begin{array}{l}\text { Reporte } \\
\text { prospectivo y } \\
\text { series de } \\
\text { casos }\end{array}$ & 28 & $\begin{array}{l}\text { USP vs. } \\
\text { radiografía y } \\
\text { TC }\end{array}$ & $\begin{array}{l}\text { El USP mostró signos de "cascada» } \\
\text { y «haz de luz» sugestivos de } \\
\text { neumonía en pacientes con } \\
\text { COVID-19; la radiografía fue inferior, } \\
\text { mientras que la TC no reveló } \\
\text { cambios pleurales relevantes }\end{array}$ & $\begin{array}{l}\text { El USP puede ayudar al } \\
\text { diagnóstico de la } \\
\text { neumonía por COVID-19, } \\
\text { superior a la radiografía y } \\
\text { TC en los cambios } \\
\text { tempranos }\end{array}$ & 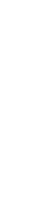 \\
\hline $\begin{array}{l}\text { Chen, et al., } \\
2020^{22}\end{array}$ & $\begin{array}{l}\text { Reporte } \\
\text { prospectivo }\end{array}$ & 51 & TC vs. USP & $\begin{array}{l}\text { El USP se consideró un patrón B, } \\
\text { línea pleural irregular y } \\
\text { consolidaciones subpleurales. Los } \\
\text { cambios pleurales no fueron visibles } \\
\text { por TC }\end{array}$ & $\begin{array}{l}\text { El USP es equiparable a la } \\
\text { TC para detectar } \\
\text { alteraciones del } \\
\text { parénquima pulmonar en } \\
\text { pacientes con COVID-19 }\end{array}$ & 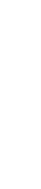 \\
\hline $\begin{array}{l}\text { Zieleskiewicz, } \\
\text { et al., } 2020^{23}\end{array}$ & $\begin{array}{l}\text { Observacional } \\
\text { multicéntrico }\end{array}$ & 100 & $\begin{array}{l}\text { USP vs. } \\
\text { radiografía }\end{array}$ & $\begin{array}{l}\text { El USP se asoció significativamente } \\
\text { con la gravedad de la neumonía } \\
\text { evaluada por la imagen de tórax y } \\
\text { las características clínicas }\end{array}$ & $\begin{array}{l}\text { El USP puede predecir la } \\
\text { severidad de la neumonía }\end{array}$ & 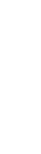 \\
\hline $\begin{array}{l}\text { Kulkarni, et al., } \\
2020^{24}\end{array}$ & $\begin{array}{l}\text { Reporte } \\
\text { prospectivo, } \\
\text { abierto antes } \\
\text { y después }\end{array}$ & 2 & $\begin{array}{l}\text { USP } \\
\text { vS. } \\
\text { radiografía }\end{array}$ & $\begin{array}{l}\text { EI USP mostró engrosamiento e } \\
\text { interrupción pleural, que no se } \\
\text { aprecia por radiografía }\end{array}$ & $\begin{array}{l}\text { El USP tiene alta } \\
\text { sensibilidad para las } \\
\text { manifestaciones } \\
\text { pulmonares de COVID-19, } \\
\text { como SDRA y } \\
\text { consolidación }\end{array}$ & 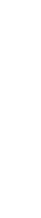 \\
\hline $\begin{array}{l}\text { Bonadia, et al., } \\
2020^{25}\end{array}$ & $\begin{array}{l}\text { Reporte } \\
\text { prospectivo }\end{array}$ & 96 & $\begin{array}{l}\text { USP vs. } \\
\text { radiografía }\end{array}$ & $\begin{array}{l}\text { EI USP es capaz de detectar la } \\
\text { neumonía por COVID-19 y predecir, } \\
\text { durante la primera evaluación } \\
\text { pacientes en riesgo de ingreso en } \\
\text { unidad de cuidados intensivos y } \\
\text { muerte }\end{array}$ & $\begin{array}{l}\text { El USP torácico puede ser } \\
\text { una herramienta para } \\
\text { predecir el pronóstico de } \\
\text { los pacientes con } \\
\text { COVID-19; es superior a la } \\
\text { radiografía para } \\
\text { establecer diagnóstico y } \\
\text { pronóstico }\end{array}$ & 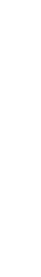 \\
\hline $\begin{array}{l}\text { Convissar, et al., } \\
2020^{26}\end{array}$ & $\begin{array}{l}\text { Reporte } \\
\text { prospectivo, } \\
\text { abierto, antes } \\
\text { y después }\end{array}$ & 43 & $\begin{array}{l}\text { USP vs. } \\
\text { radiografía }\end{array}$ & $\begin{array}{l}\text { El USP mostró lesiones en el campo } \\
\text { pulmonar posterior e inferior, líneas } \\
\mathrm{B} \text {, líneas pleurales distorsionadas, } \\
\text { consolidaciones pulmonares } \\
\text { subpleurales y broncogramas aéreos }\end{array}$ & $\begin{array}{l}\text { La TC puede ser inferior al } \\
\text { USP para detectar } \\
\text { lesiones y derrames } \\
\text { periféricos más pequeños } \\
\text { en pacientes con } \\
\text { COVID-19 }\end{array}$ & 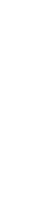 \\
\hline
\end{tabular}


Tabla 1. Resumen de los resultados por estudio, se enlista autor (año), número de pacientes, comparador, desenlace y conclusión de cada publicación (Continuación)

\begin{tabular}{|c|c|c|c|c|c|}
\hline Autor, año & $\begin{array}{l}\text { Tipo de } \\
\text { estudio }\end{array}$ & Pacientes & Comparados & Desenlace & Conclusión \\
\hline $\begin{array}{l}\text { Buda, et al., } \\
2020^{27}\end{array}$ & $\begin{array}{l}\text { Reporte } \\
\text { prospectivo y } \\
\text { series de } \\
\text { casos }\end{array}$ & 4 & $\begin{array}{l}\text { USP vs. } \\
\text { radiografía }\end{array}$ & $\begin{array}{l}\text { EI USP mostró datos sugestivos de } \\
\text { neumonía intersticial, mientras que } \\
\text { la radiografía no era un } \\
\text { diferenciador por imagen }\end{array}$ & $\begin{array}{l}\text { El USP muestra datos } \\
\text { tempranos y puede ayudar } \\
\text { a la toma de decisiones }\end{array}$ \\
\hline $\begin{array}{l}\text { Abrams, et al., } \\
2020^{28}\end{array}$ & $\begin{array}{l}\text { Reporte } \\
\text { prospectivo y } \\
\text { series de } \\
\text { casos }\end{array}$ & 42 & $\begin{array}{l}\text { USP vs. } \\
\text { radiografía }\end{array}$ & $\begin{array}{l}\text { EI USP seriado mostró progresión de } \\
\text { la COVID-19 es el parénquima } \\
\text { pulmonar, líneas B dispersas, a las } \\
\text { líneas B confluyentes con } \\
\text { consolidación; estos hallazgos no } \\
\text { fueron evaluable por radiografía }\end{array}$ & $\begin{array}{l}\text { El USP es superior en el } \\
\text { diagnóstico de casos } \\
\text { leves y moderados que no } \\
\text { tienen cambios por } \\
\text { radiografía }\end{array}$ \\
\hline $\begin{array}{l}\text { Sultan, et al., } \\
2020^{29}\end{array}$ & $\begin{array}{l}\text { Reporte } \\
\text { prospectivo y } \\
\text { series de } \\
\text { casos }\end{array}$ & 69 & $\begin{array}{l}\text { USP vs. } \\
\text { radiografía }\end{array}$ & $\begin{array}{l}\text { EI USP mostró que los patrones } \\
\text { comunes observados son } \\
\text { bilaterales, predominio posterobasal } \\
\text { y múltiples líneas B que van desde } \\
\text { focal a difusa }\end{array}$ & $\begin{array}{l}\text { El USP supera a la } \\
\text { radiografía de tórax en el } \\
\text { diagnóstico de afección } \\
\text { pleural y del parénquima } \\
\text { pulmonar en COVID-19 }\end{array}$ \\
\hline $\begin{array}{l}\text { Zhao, et al., } \\
2020^{30}\end{array}$ & $\begin{array}{l}\text { Estudio de } \\
\text { corte } \\
\text { transversal, } \\
\text { observacional } \\
\text { prospectivo }\end{array}$ & 35 & USP vs. TC & $\begin{array}{l}\text { El USP mostró abundantes líneas B, } \\
\text { patrones de consolidación y } \\
\text { derrame pleural, mientras que la TC } \\
\text { mostró vidrio despulido, } \\
\text { consolidaciones y derrame pleural }\end{array}$ & $\begin{array}{l}\text { Los hallazgos de COVID-19 } \\
\text { a nivel pulmonar por USP } \\
\text { y TC son equiparables, el } \\
\text { USP puede ser una } \\
\text { herramienta para el } \\
\text { diagnóstico a la cabecera } \\
\text { del paciente }\end{array}$ \\
\hline $\begin{array}{l}\text { Cioni, et al., } \\
2020^{31}\end{array}$ & $\begin{array}{l}\text { Reporte de } \\
\text { caso } \\
\text { prospectivo }\end{array}$ & 10 & $\begin{array}{l}\text { USP vs. } \\
\text { radiografía }\end{array}$ & $\begin{array}{l}\text { EI USP fue capaz de reconocer } \\
\text { signos de afectación pulmonar } \\
\text { inicial incluso en pacientes con } \\
\text { enfermedad leve, mostrando líneas } \\
\text { B no confusas esporádicas, } \\
\text { consolidaciones subpleurales poco } \\
\text { frecuentes y engrosamiento pleural } \\
\text { esporádico; la radiografía no mostró } \\
\text { datos tempranos }\end{array}$ & $\begin{array}{l}\text { EI USP es superior a la } \\
\text { radiografía para definir el } \\
\text { daño pulmonar }\end{array}$ \\
\hline $\begin{array}{l}\text { Møller-Sørensen, } \\
\text { et al., } 2020^{32}\end{array}$ & $\begin{array}{l}\text { Observacional } \\
\text { multicéntrico }\end{array}$ & 10 & $\begin{array}{l}\text { USP vs. } \\
\text { radiografía }\end{array}$ & $\begin{array}{l}\text { EI USP mostró cambios periféricos } \\
\text { en el tejido pulmonar, los cambios } \\
\text { severos fueron consolidaciones y } \\
\text { una línea pleural engrosada. Las } \\
\text { líneas B eran multifocales o } \\
\text { confluyentes; la radiografía no } \\
\text { muestra cambios pleurales ni muy } \\
\text { periféricos }\end{array}$ & $\begin{array}{l}\text { Usando una puntuación } \\
\text { para los cambios } \\
\text { pulmonares, el USP se } \\
\text { puede usar para distinguir } \\
\text { a los pacientes con } \\
\text { mejoría de la función } \\
\text { pulmonar de los pacientes } \\
\text { con función pulmonar } \\
\text { estacionaria o deteriorada }\end{array}$ \\
\hline
\end{tabular}

USP: ultrasonido pulmonar; COVID-19: enfermedad por coronavirus 2019; TC: tomografía computarizada; SDRA: síndrome de dificultad respiratoria aguda.

a gran escala; encontraron imágenes ultrasonográficas de pacientes con SARS-CoV-2 donde el $86 \%$ mostraron opacidad de vidrio esmerilado, el $76 \%$ distribución bilateral y el 33\% distribución periférica $^{7,20,23}$. Smith observó que el uso de radiografías de tórax portátiles también plantea el problema de la contaminación, a menos que se reserve una máquina dedicada para las áreas de reconversión ${ }^{36}$, sin embargo, la misma limitación se presenta en el equipo de ultrasonido y TC. Varios autores concluyen que la radiografía de tórax se correlaciona poco con el cuadro clínico cuando se compara con el USP21,22,24,25,37. Chen estudió a 51 pacientes comparando el USP con la radiografía de tórax y demostró poca correlación entre la especificidad de ambas técnicas de imagen para la detección de COVID-19, también encontró que los hallazgos radiológicos compatibles con COVID-19 estuvieron presentes en 37 pacientes $(72.5 \%)$ en la TC y 40 pacientes $(78.4 \%)$, con sensibilidad del $100.0 \%$, especificidad del $78.6 \%$, 


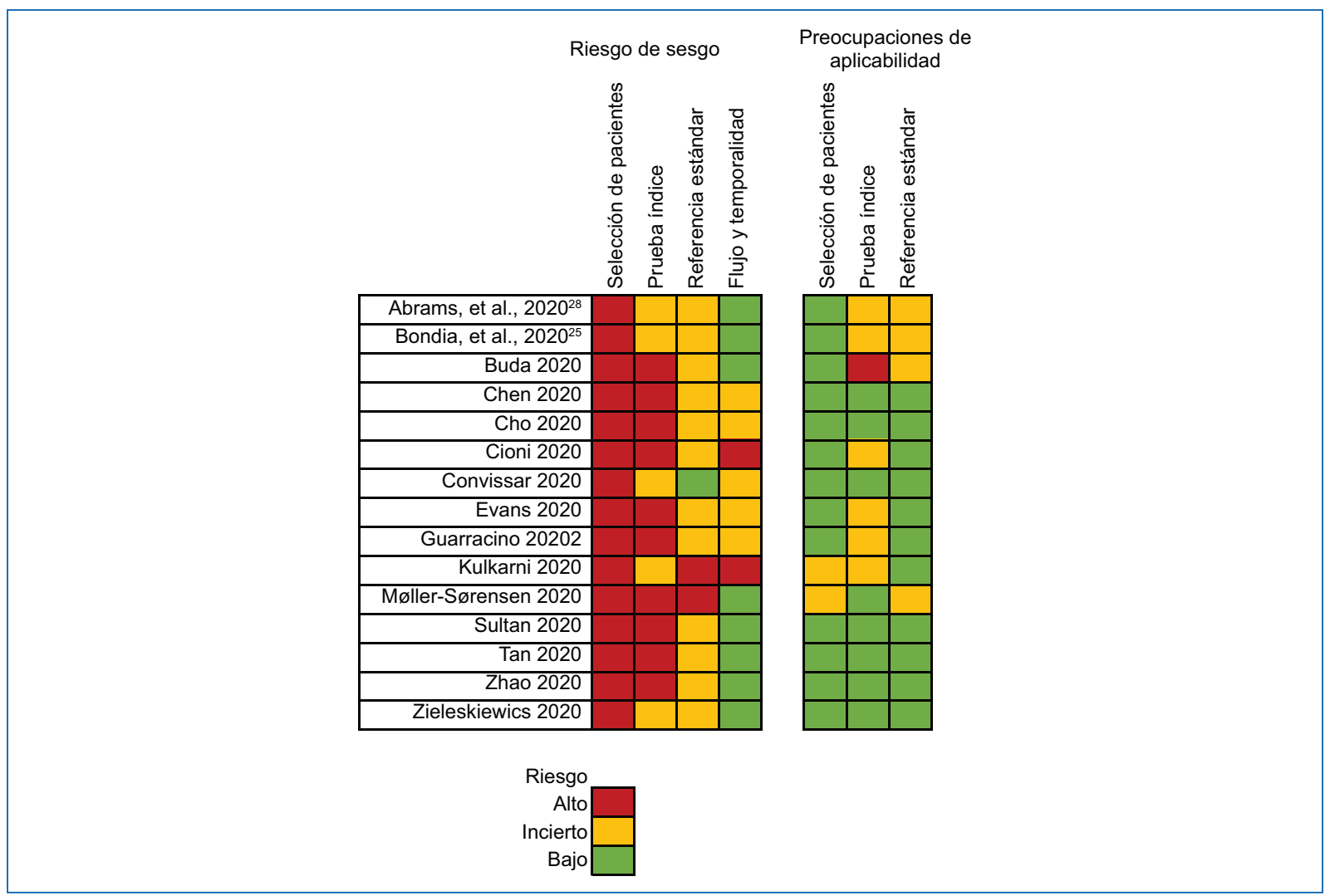

Figura 2. Gráfica de la calidad metodológica de los estudios incluidos.

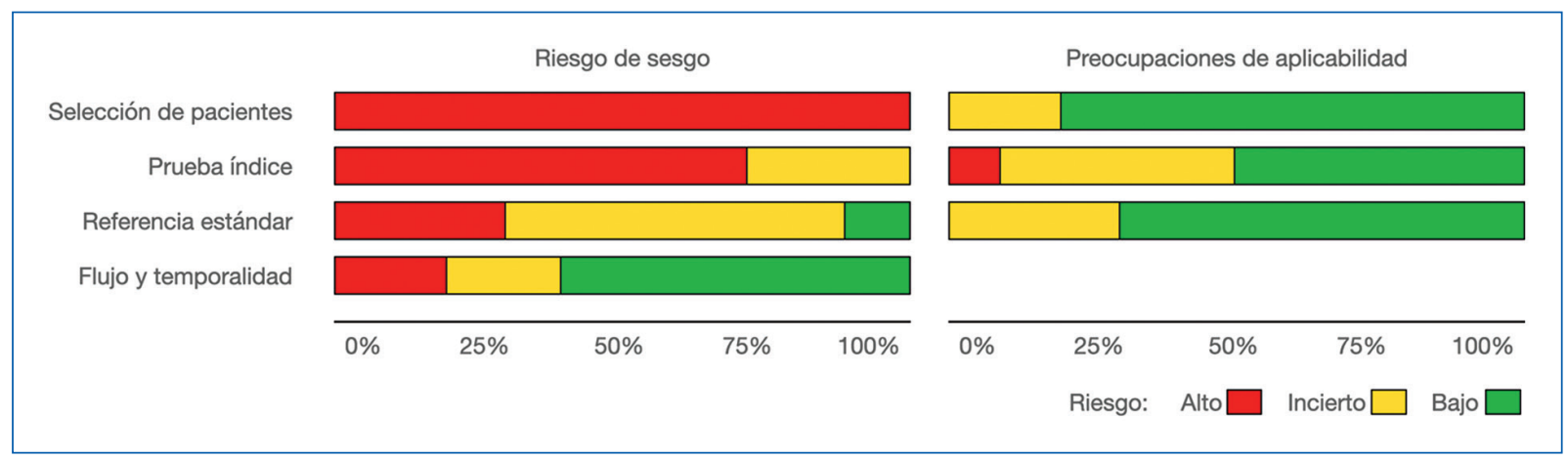

Figura 3. Resumen de la calidad metodológica de los estudios incluidos.

valor predictivo positivo del $92.5 \%$ y negativo del $100.0 \%$ para el USP, mostrando alta correlación entre los hallazgos y anormalidades asociadas a este virus utilizando ambas técnicas ${ }^{22}$. Cho concluyó que, aunque la TC de tórax es un método útil para diagnosticar COVID-19, sin embargo, el riesgo de contagio y la exposición a la radiación han obstaculizado el uso sistemático de las modalidades de diagnóstico como la TC ${ }^{8}$.

\section{Fortalezas y limitaciones}

Se trató de una revisión sistemática de la utilidad del USP, un estudio que aún no es un estándar de atención y del que se cuenta con poca información en comparación a otros métodos de imagen torácica; además relacionado con una enfermedad nueva, con manifestaciones pleuropulmonares variadas. Existen pocas publicaciones, escasas revisiones sistemáticas (la 
mayoría solo incluyen reportes de caso, series de casos y pequeñas cohortes), por lo que los autores consideramos que la evidencia, resultados y conclusiones pueden cambiar con el tiempo y el incremento de las publicaciones relacionadas con el tema. Se asume que los autores de los diferentes trabajos revisados tienen adecuado entrenamiento y praxia en la realización del USP, pero solo algunos trabajos reportaron el tipo de transductor, configuración del software y la técnica de rastreo con el transductor, lo que puede explicar los diferentes hallazgos entre los centros.

\section{Conclusiones}

El USP es una herramienta útil para el diagnóstico de COVID-19. Puede brindar otros datos relevantes para establecer diagnósticos diferenciales y pautas de manejo, y puede realizarse de forma seriada a la cabecera del paciente, lo que ayuda al seguimiento, reduce la exposición del personal, traslado y radiación, cuando se compara frente a la radiografía de tórax y la TC.

\section{Financiamiento}

La presente investigación no ha recibido ninguna beca específica de agencias de los sectores públicos, comercial o sin ánimo de lucro.

\section{Conflicto de intereses}

Los autores declaran no tener conflicto de intereses.

\section{Responsabilidades éticas}

Protección de personas y animales. Los autores declaran que para esta investigación no se han realizado experimentos en seres humanos ni en animales.

Confidencialidad de los datos. Los autores declaran que en este artículo no aparecen datos de pacientes.

Derecho a la privacidad y consentimiento informado. Los autores declaran que en este artículo no aparecen datos de pacientes.

\section{Bibliografía}

1. Organización Mundial de la Salud. Coronavirus disease (COVID-19) pandemic [Internet]. Organización Mundial de la Salud; 2020 [acceso: 5 de agosto de 2020]. Disponible en: https://www.who.int/emergencies/diseases/novel-coronavirus-2019?gclid=EAlalQobChMloPeYp6r-7AIVCtvACh1ulgUOEAAYASAAEgL87PD_BwE

2. Coronavirus COVID19. Comunicado Técnico Diario [Internet]. México: Secretaría de Salud, Dirección General de Epidemiología; 2020 [acceso: 5 de agosto de 2020].. Disponible en: https://www.gob.mx/salud/ documentos/coronavirus-covid-19-comunicado-tecnico-diario-238449
3. Todo sobre el COVID-19 [Internet]. México: Gobierno de México, Secretaría de Salud; 2020 [acceso: 5 de agosto de 2020]. Disponible en: https://coronavirus.gob.mx/

4. Duclos G, Lopez A, Leone M, Zieleskiewicz L. "No dose" lung ultrasound correlation with "low dose" CT scan for early diagnosis of SARS-CoV-2 pneumonia. Intensive Care Med. 2020;46(6):1103-4.

5. Zhang H, Penninger JM, Li Y, Zhong N, Slutsky AS. Angiotensin - converting enzyme 2 (ACE2) as a SARS-CoV-2 receptor: molecular mechanisms and potential therapeutic target. Intensive Care Med. 2020;46(5):586-90.

6. Yasukawa K, Minami T. Point-of-care lung ultrasound findings in patients with COVID-19 pneumonia. AM J Trop Med Hyg. 2020;102(6):1198-202.

7. Tan G, Lian X, Zhu Z, Wang Z, Huang F, Bachelor YZ, et al. Use of lung ultrasound to differentiate COVID-19 pneumonia from community-acquired pneumonia. Ultrasound Med Biol. 2020;46(1):2651-8.

8. Cho YJ, Song KH, Lee Y, Yoon JH, Park JY, Jung J, et al. Lung ultrasound for early diagnosis and severity assessment of pneumonia in patients with coronavirus disease 2019. Korean J Intern Med. 2020;35(4):771-81.

9. Lichtenstein DA, Lascols N, Mezière G, Gepner A. Ultrasound diagnosis of alveolar consolidation in the critically ill. Intensive Care Med. 2004;30(2):276-81.

10. Beckmann U, Gillies DM, Berenholtz SM, Wu AW, Pronovost P. Incidents relating to the intra-hospital transfer of critically ill patients An analysis of the reports submitted to the Australian Incident Monitoring Study in Intensive Care. Intensive Care Med. 2004;30(8)1579-85.

11. Lichtenstein DA, Mezière GA. Relevance of lung ultrasound in the diagnosis of acute respiratory failure: The BLUE protocol. 2008;134(1):117-25.

12. Colmenero M, García-Delgado M, Navarrete I, López-Milena G. Utilidad de la ecografía pulmonar en la unidad de medicina intensiva. Med Intensiva. 2010;34(9):620-8.

13. Mortensen EM, Restrepo MI, Anzueto A, Pugh JA. Antibiotic therapy and 48hour mortality for patients with pneumonia. Am J Med. 2006;119(10):859-64.

14. Vignon $P$, Repessé $X$, Vieillard-baron A, Maury E. Critical care ultrasonography in acute respiratory failure. Crit Care. 2016;20(1):228.

15. Amatya Y, Rupp J, Russell FM, Saunders J, Bales B, House DR. Diagnostic use of lung ultrasound compared to chest radiograph for suspected pneumonia in a resource-limited setting. Int J Emerg Med. 2018;11(1):8.

16. Brenner DJ, Hall EJ. Computed tomography - An increasing source of radiation exposure. N Eng J Med. 2007;357(22):2277-84.

17. Liu X, Lian R, Tao Y, Gu C, Zhang G. Lung ultrasonography: an effective way to diagnose community-acquired pneumonia. Emerg Med J. 2015;32(6):433-8.

18. Lichtenstein DA, Mezière GA. The BLUE-points: three standardized points used in the BLUE-protocol for ultrasound assessment of the lung in acute respiratory failure. Crit Ultrasound J. 2011;3:109-10.

19. Lichtenstein DA. BLUE-Protocol and FALLS-Protocol: Two applications of lung ultrasound in the critically ill. Chest. 2015;147(6):1659-70.

20. Evans KD, Yang Q, Liu Y, Ye R, Peng C. Sonography of the lungs: Diagnosis and surveillance of patients with COVID-19. J Diagnostic Med Sonogr. 2020;36:370-6.

21. Guarracino F, Vertugno L, Forfori F, Corradi F, Orso D, Bertini P, et al. Lung, heart, vascular, and diaphragm ultrasoun examination of COVID-19 patients: a comprehensive approach. J Cardiothorac Vasc Anesth. 2020 Jun 11;S10530770(20)30519-X. doi: 10.1053/j.jvca.2020.06.013. Online ahead of print.

22. Chen YT, Martí de García M, Díez-Tazcón A, Alonso-González R, Agudo-Fernández S, Parra-Gordo ML, et al. Correlation between chest computed tomography and lung ultrasonography in patients with coronavirus disease 2019 (COVID-19). Ultrasound Med Biol. 2020;46(11):2918-26.

23. Zieleskiewicz L, Markarian T, Lopez A, Taguet C, Mohammedi N, Boucekine $\mathrm{M}$, et al. Comparative study of lung ultrasound and chest computed tomography scan in the assessment of severity of confirmed COVID - 19 pneumonia. Intensive Care Med. 2020;46(9):1707-13.

24. Kulkarni S, Down B, Jha S. Point-of-care lung ultrasound in intensive care during the COVID-19 pandemic. Clin Radiol. 2020;75(9):710e1-710e4.

25. Bonadia N, Carnicelli A, Piano A, Buonsenso D, Gilardi E, Kadhim C, et al. Lung ultrasound findings are associated with mortality and need of intensive care admission in COVID-19 patients evaluated in the Emergency Department. Ultrasound Med Biol. 2020;46(11):2927-37.

26. Convissar D, Gibson LE, Berra L, Bittner EA, Chang MG. Application of lung ultrasound during the coronavirus disease 2019 pandemic: A narrative review. Anesth Analg. 2020;131(2):345-50.

27. Buda N, Segura-Grau E, Cylwik J, Wełnicki M. Lung ultrasound in the diagnosis of COVID-19 infection - A case series and review of the literature. Adv Med Sci. 2020;65(2):378-85.

28. Abrams ER, Rose G, Fields JM, Esener D. Point-of-care ultrasound in the evaluation of COVID-19. J Emerge Med. 2020;59(3):403-8.

29. Sultan LR, Sehgal CM. A review of early experience in lung ultrasound in the diagnosis and management of Covid-19. Ultrasound Med Biol. 2020;46(9):2530-45.

30. Zhao L, Yu K, Zhao Q, Tian R, Xie H, Xie J, et al. Lung ultrasound score in evaluating the severity of coronavirus disease 2019 (COVID-19) pneumonia. Ultrasound Med Biol. 2020;46(11):2938-44.

31. Cioni G, Bellandi G, Bertolini S, Betti G, Bialek W, Fedeli A, et al. A multisystem approach by bed-side ultrasound in patients with COVID-19 infection: a case series. Ital J Med. 2020;2020;14:106-11. 
32. Møller-Sørensen H, Gjedsted J, Jørgensen VL, Lindskov Hansen K. COVID-19 assessment with bedside lung ultrasound in a population of intensive care patients treated with mechanical ventilation and ECMO. Diagnostics (Basel). 2020;10(7):447.

33. Huang $\mathrm{Y}$, Wang S, Liu Y, Zhang Y, Zheng C, Zheng Y, et al. A preliminary study on the ultrasonic manifestations of peripulmonary lessions of non-critical novel Coronavirus pneumonia (COVID-19) [Internet]. SSRN 2020 [acceso 10 de agosto de 2020]. Disponible en: https://poseidon01 ssrn.com/delivery.phplD=868 1030030641141151231020690750970920 $60 \quad 0703701005302510007208502602910007100102310806304303506$ 0330441071150280930910911070030570480870110590120871151210 0910890980600660870741070050190310990831161061070220850281 1012124127066115108107071100105100002\& EXT=pdf\&INDEX=TRUE
34. Peng QY, Wang XT, Zhang LN. Findings of lung ultrasonography of novel coronavirus pneumonia during the 2019-2020 epidemic. Intensive Care Med. 2020;46(5):849-50.

35. COVID-19 rapid guideline: critical care in adults [Internet]. National Institute for Health and Care Excellence; 2020 [acceso: 10 de agosto de 2020]. Disponible en: https://www.nice.org.uk/guidance/ng159

36. Smith MJ, Hayward SA, Innes SM, Miller ASC. Point-of-care lung ultrasound in patients with COVID-19 - a narrative review. Anaesthesia. 2020;75(8):1096-104.

37. Fraile Gutiérrez V, Ayuela Azcárate JM, Pérez-Torres D, Zapata L, Rodríguez Yakushev A, Ochagaía A. Ecografía en el manejo del paciente crítico con infección por SARS-CoV-2 (COVID-19): una revisión narrativa. Med Intensiva. 2020;44(9):551-65. 